\title{
Client-side Energy Costs of Video Streaming
}

\author{
Oche Ejembi \\ School of Computer Science \\ University of St Andrews, Fife, UK \\ Email: ooe@st-andrews.ac.uk
}

\author{
Saleem N. Bhatti \\ School of Computer Science \\ University of St Andrews, Fife, UK \\ Email: saleem@st-andrews.ac.uk
}

\begin{abstract}
Through measurements on our testbed, we show how users of Netflix could make energy savings of up to $34 \%$ by adjusting video quality settings. We estimate the impacts of these quality settings on the energy consumption of client systems and the network. If users exercise choice in their video streaming habits, over $100 \mathrm{GWh}$ of energy a year could be saved on a global scale. We discuss how providing energy usage information to users of digital video could enable them to make choices of video settings to reduce energy usage, and we estimate savings on associated electricity costs and carbon emissions.
\end{abstract}

\section{INTRODUCTION}

Video-on-Demand (VoD) is by far the most popular type of traffic on the Internet and its use is growing. According to the Sandvine Global Internet Phenomena report for 1H 2014 [1], VoD was responsible for $64 \%$ of all the downstream traffic experienced on fixed networks, and $40 \%$ on mobile networks in the US, with similarly large proportions experienced in Europe, Asia-Pacific, and Latin America. Cisco estimates that by $2018,79 \%$ of all the world's Internet traffic will be some form of video [2].

However, video consumes more resources - network capacity, device CPU utilisation, memory, I/O, disk space etc. - than the other popular uses of the Internet, such as text, audio and still images. So, video also consumes more energy.

\section{A. Contributions and Structure}

We measure the client-side energy usage of the popular video-on-demand service provider Netflix to make global Fermi estimates of how much energy could be consumed by users of this particular service. We make suggestions on how this energy usage could be reduced or made more sustainable. Our contributions are:

1) Using a simple measurement methodology, we make an empirical assessment of the client-side energy usage of the world's most popular premium VoD provider. We then make global estimates of how much energy is consumed by this service's subscribers.

2) We examine the network characteristics of this video application and investigate the energy impacts of the traffic at the client side.

3) Finally, we discuss ways in which these users could be empowered to make choices towards saving energy while using VoD services, based on measured energy usage information, and feedback to the users.

In Section II, we discuss recent, related work. This is followed by a presentation of our experiment methodology, testbed and measurement strategies in Section III, with the results from our experiments in Section IV. We analyse the results in Section $\mathrm{V}$, including a discussion of the implications for real-world scenarios in Section VI. Finally, we conclude and present avenues for future work in Section VII.

\section{RELATED WORK}

Research in Green ICT and energy efficient systems now covers a wide range of topics. There have been high-level, analytical investigations of ICT energy usage e.g. Somavat et el. [3] for energy-efficient data- centres and servers [4], to user studies towards reducing energy usage in ICT [5].

Considering the scale of Internet traffic attributed to video, a surprisingly small amount of work has specifically looked at energy usage of multimedia, especially for non-mobile systems. Non-mobiles are responsible for the bulk of video traffic $-60 \%$ according to Nielsen [6] - and so also for the associated energy use and carbon emissions. In our previous work [7], we performed an empirical investigation into the energy usage for video decoding and encoding for different popular codecs on a commodity desktop hardware system. We observed a difference of up to a factor of 3 between software codecs for decoding, even at the same picture size (resolution) and bit rate, for our desktop client system. Similarly, Shehabi et al. [8], make an analytical life-cycle assessment of the energy used by streaming video in the US. They identify that the strongest opportunities for significant gains in energy efficiency are at the end-user level and the network.

A considerable amount of research has investigated various aspects of popular multimedia applications like Netflix, YouTube and BBC iPlayer, similar (in principle) to our focus in this paper e.g [9] and [10]. However, very little work has focused explicitly on the energy usage of these services and applications, and the few that do consider such issues focus on mobile devices with the aim of extending battery life [11].

We take the position that since the bulk of video traffic is consumed by non-mobile devices (according the Sandvine report [1] and Nielsen [6]), it is important to investigate energy usage of non-mobiles, especially as those systems will not have the same level of design or engineering optimisation for energy efficient operation. As the popularity of $\mathrm{VoD}$ continues to increase, so will the energy cost and any associated carbon emissions. However, where mains power is easily available, there is the danger that energy consumption by VoD could 
become a large drain on future energy usage, which would impact energy prices and energy supply.

\section{Methodology}

The aim of our experiment was to perform an empirical investigation of the energy, bandwidth and resource consumption of Netflix during normal playback operations. Based on that energy usage, we have made estimates of the energy consumption of desktop VoD users and how much energy could be saved if users exercise choice in their use of video.

\section{A. Testbed}

The testbed is shown Figure 1, with hardware similar to that used for our previous work [7]. For each experiment run, a single client host was used to playback videos streamed from Netflix - two of these units are shown in the left of the Figure 1. Each system is a Shuttle XPC Glamor SG31G2 with an Intel $\AA$ Core $^{\mathrm{TM}} 2$ Quad Q6600, 2.40GHz CPU, Intel 82G33/G31 chipset using an Express Integrated Graphics Controller core, with 4GB DRAM (128MB used for graphics, configured in the BIOS). To examine the effects of hardwareassisted video decoding, we performed experiments with a PCI-express Nvidia $\left(\mathrm{B}\right.$ G86 GeForce ${ }^{\mathrm{TM}} 8400 \mathrm{GS}$ with $256 \mathrm{MB}$ GDRAM housed in one of the systems. Both clients were connected to the Internet using $1 \mathrm{Gbps}$ Ethernet links to the University's Joint Academic Network (JANET) connection, the UK NREN. This connectivity offers download rates that are well above the UK household average - a download rate of $600 \mathrm{Mbps}$ has been observed, compared to the UK household average in 2014 of $18.7 \mathrm{Mbps}$ [12]. So we were able to observe Netflix traffic in near ideal network conditions (little or no capacity bottleneck and/or congestion on the end-to-end path).

The client hosts ran a minimal installation of Ubuntu Linux (v13.10 64-bit x86-64 server), and with a minimal set of background processes running. This was to avoid any unnecessary load on the machines when measuring energy usage. We used the lightweight Openbox window manager, and the Mozilla Firefox (v29.0) web browser with the Pipelight plugin (v0.2.6, a Linux port of Silverlight, version 0.2.6) for playback of video content from the Netflix UK website. We measured energy usage through a Kill-a-Watt power meter ${ }^{1}$, modified to send power readings via a short-range radio link to a USB receiver on the experiment controller at 1-second intervals.

\section{B. Measurement regime}

The Netflix homepage presents a wide selection of titles which a user may be interested in. These videos are selected using Netflix's recommender algorithm, which takes into consideration user preferences and viewing history. We were presented with 202 unique titles for viewing. To gain a wide and representative sample space, we decided to run our experiments on them all. On personal computers/laptops users may select one of three preferred quality levels (LOW, MEDIUM and HIGH), or may allow Netflix to automatically

\footnotetext{
${ }^{1}$ http://www.p3international.com/products/p4400.html
}

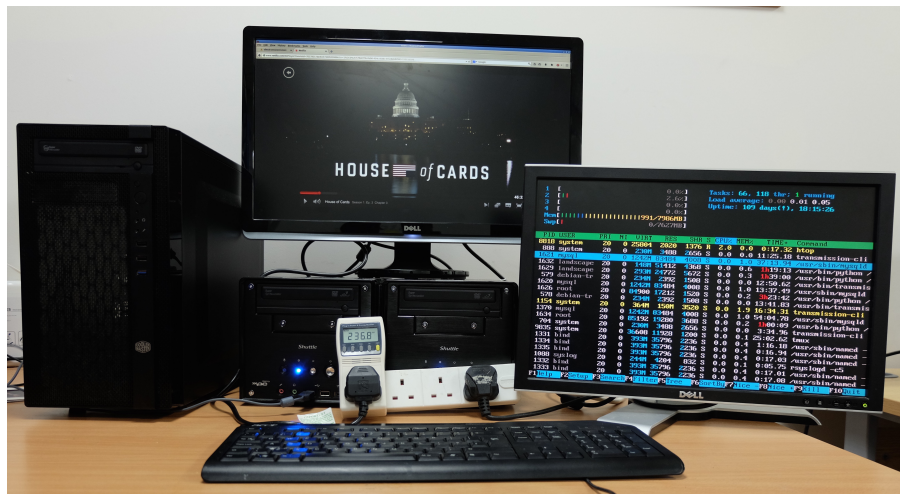

Fig. 1: Our video playback testbed. The black Shuttle systems (centre) were used for the playback experiments, and are connected to the Kill-AWatt/MoteWatt power meter (centre-left, just above the keyboard). The mini-tower on the left is the experiment-controller, not part of the system being measured. The energy consumption of the display units was not measured: one shows the video being played back (centre), the other shows part of the measurement system (right).

choose based on current network conditions. This quality selection page is shown in Figure 2.

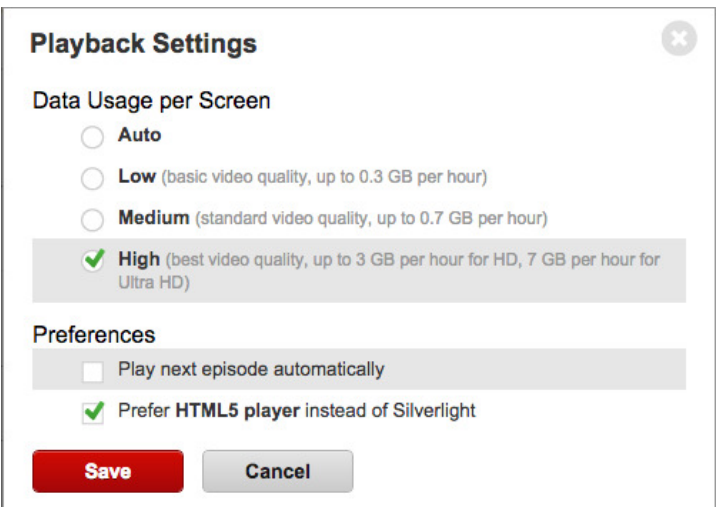

Fig. 2: Netflix playback quality settings for selection by the user.

For each of the 202 titles and at each of the 3 quality levels, we streamed 2 minutes of video and repeated this 5 times. The starting point for playback for each title was randomly chosen for each video, but remained constant for that particular title. For example, for all the runs for the title 'House of Cards (episode 1)', we started streaming all 5 runs from the 00:19:00 time mark to the 00:21:00 time mark, but for 'Orange is the New Black', we streamed from 00:05:00 to 00:07:00, and so on. Netflix tries to save the point at which a video is paused or stopped, so that playback may be resumed from that point. While this feature enhances user experience, it would have perturbed experimental repeatability and reproducibility. So, we implemented a simple firewall rule to drop Netflix control messages (which are sent via HTTP) matching the regular expression '*nccp/controller*', effectively disabling this behaviour. For each run, we sampled the power usage (with the Kill-A-Watt meter), CPU and memory utilization (with top), and network usage (with tshark) every 1 second. For 202 titles and at 3 quality levels, repeated 5 times, giving a total of over 3030 minutes of measurements. 


\section{Results}

We captured the CPU utilisation, memory usage, network usage and power consumption at 1 second intervals. In Figure 3 , we present summary results from the entire corpus. This shows, as expected, that there is a difference (comparing medians) in energy usage, CPU utilisation and network usage, between LOW, MEDIUM and HIGH quality playback. However, given the diversity of content, there are overlaps at the extreme ranges of values for LOW, MEDIUM and HIGH. For example, some HIGH quality streams use less energy than some LOW quality streams (Figure 3a).

\section{A. Energy usage metric for video}

Previously (see [7]), we have defined energy metrics for video. $P_{d v}$ represents the amount of energy used to decode/playback a second of a given video on a given system:

$$
\begin{aligned}
P_{d v} & =\frac{\text { energy usage for video decoding/playback }}{t_{v}} \\
& =P_{a}-P_{\text {idle }}
\end{aligned}
$$

where $P_{d v}$ is the mean energy usage per second of video $\left(J / s_{v}\right)$ by video decoding/playback $\left(P_{d v}\right)$, and $t_{v}$ is the duration of the video stream in seconds. $P_{a}$ is the mean measured power of the system during encoding or decoding of the video. $P_{\text {idle }}$ is the mean measured power when the system lies idle, as summarised in Table I. The units Joules per second (of video content), i.e. $J / s_{v}$ are used instead of Watts $(\mathrm{W}=$ $J / s$ ) to make clear the different assessment we are making, and to allow comparison directly with a similar metric, $P_{e v}$, for encoding video, even when encoding is not real-time.

TABLE I: Definitions for $P_{d v}$ (Eqn. (1)).

\begin{tabular}{|c|l|}
\hline$P_{d v}$ & mean energy usage per second for video decoding $\left[\mathrm{J} / \mathrm{s}_{v}\right]$ \\
\hline$P_{a}$ & measured mean system power usage during video playback [W] \\
\hline$P_{i d l e}$ & mean system power usage when idle [W] \\
\hline$t_{v}$ & duration of video sample [s] \\
\hline
\end{tabular}

\section{B. Energy Usage}

We captured the system wide energy usage when playing back video streams at the three available video qualities. This energy usage was a system-wide measurement, the result of a combination of several individual components of the computer such as the CPU, the memory and the network interface. We chose to investigate the energy use of system as a whole as we argue that this would be more relevant and significant to users / applications trying to be energy-aware: this is the real energy usage seen by the user when the application is running. However, different system components will have different energy usage (see Section IV-C).

In Figure 3a, we present standard boxplots of the mean energy used in video playback $\left(P_{d v}\right)$ at the three available quality levels (i.e. LOW, MEDIUM and HIGH). This chart is a summary of our entire experiment's corpus of 202 Netflix videos, grouped by the three quality levels. Each data point used for the boxplot is the mean energy used in decoding (as calculated by equation 1) of 5 repetitions of a 2-minute playback of a single video title. We obtain average values of $10.8 \mathrm{~J} / s_{v}, 12.7 \mathrm{~J} / s_{v}$ and $14.5 \mathrm{~J} / \mathrm{s}_{v}$ for the respective quality levels. This corresponds to a difference of $34 \%$ between LOW and HIGH quality levels.

From Figure 3, we observe some significant variability in the energy and system resource usage over the entire corpus, even at the same quality levels. In Figure 4, we make a more fine-grained analysis of the corpus. We further group the energy usage data by genre. This highly-specific genre classification system is used by Netflix as part of its recommendation mechanism, e.g. a subscriber interested in SciFi and Fantasy films is more likely to watch other films in this genre (or a closely-related one) and as such will be recommended similar movies. The aim of this particular exercise was to investigate whether the general spatial and temporal characteristics of these genre have an effect on the overall energy and system resource used by the various genres. The only definite trend we observe in this case is the difference observed in grouping by quality level across the genres. This is because the genre system used by Netflix is based more on subjective classification of the artistic content of the video titles. However, future work could make a more detailed investigation of the relationship between the spatiotemporal characteristics of videos and energy/system resource usage. In Table II, we list the videos which consumed the least (best-case) and the most (worst-case) energy usage $\left(P_{d v}\right)$. The values are the mean of 5 runs, with a $95 \%$ confidence interval that is less than $1 \mathrm{~J} / s_{v}$ to adjust for experimental variation.

\begin{tabular}{|l|l|l|l|}
\hline Quality & Title & Genre & $P_{d v}$ \\
\hline \multirow{2}{*}{ Low } & 'The Hobbit (Part 1)' & AAA & 7.9 (Best) \\
\cline { 2 - 4 } & 'Parade's End' & BRF & 12.8 (Worst ) \\
\hline \multirow{2}{*}{ Medium } & 'Would You Rather' & HRF & 9.8 (Best) \\
\cline { 2 - 4 } & 'Fresh Meat (S1E1)' & BRT & 15.9 (Worst) \\
\hline \multirow{2}{*}{ High } & 'Peter Pan' & CFF & 9.7 (Best) \\
\cline { 2 - 4 } & 'Toy Story' & CFF & 17.3 (Worst) \\
\hline
\end{tabular}

TABLE II: Comparison of the videos with the best (lowest) and worst (highest) energy usage metrics.

\section{System resource usage}

We measured the usage of system resources including CPU and memory utilisation, with the Unix process monitoring tool top, and the network bandwidth utilisation, with the opensource network monitoring tool tshark. In Figure $3 \mathrm{~b}$ and $3 \mathrm{c}$, we show boxplots summarising the CPU utilisation and network bitrate for the entire corpus, grouped by Netflix quality level. Each data point is the mean value for the resource used from 5 repetitions of playback of a video title.

The usage of CPU, memory and network show a varying degree of correlation with overall energy usage as shown in Figure 7. Remember that we measure overall energy usage: while CPU usage shows the strongest correlation with the overall system power, with a correlation coefficient of 0.89 , examining the correlation for memory usage and mean bitrate with overall energy usage, it would be easy to draw the erroneous conclusion that those resources account for energy usage as much as CPU usage does. However, several studies 


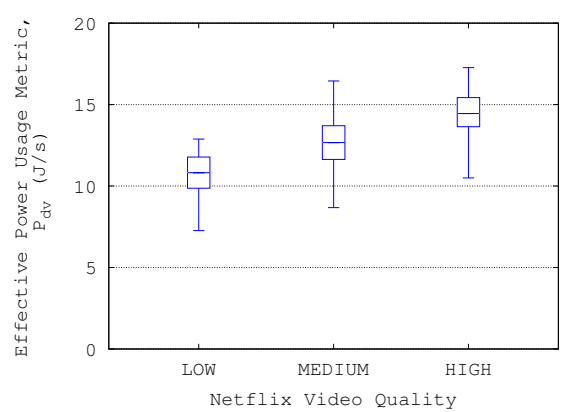

(a) Mean power usage

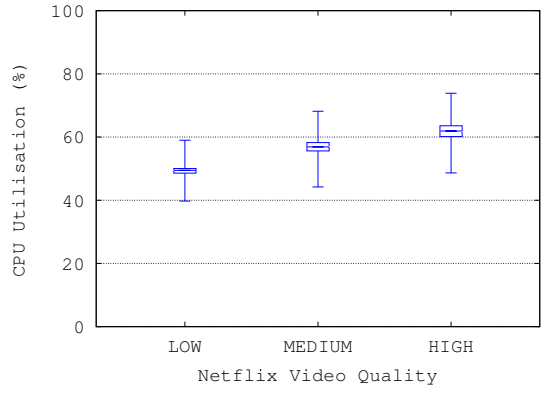

(b) Mean CPU utilisation

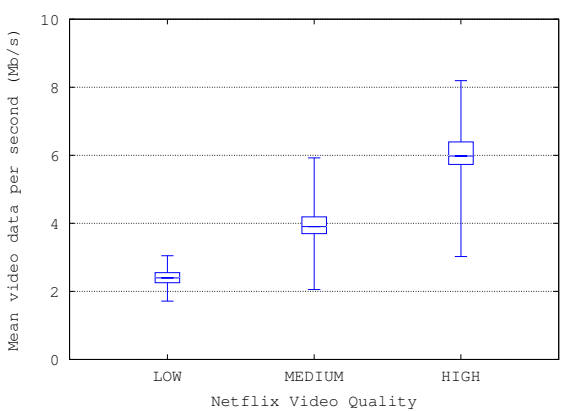

(c) Mean bitrate

Fig. 3: Summary of the resource usage (energy, CPU and network) for playback of the entire corpus of Netflix videos at the available quality levels. ( 202 videos. Each data point is the mean of 5 runs, each run is 120 seconds.)

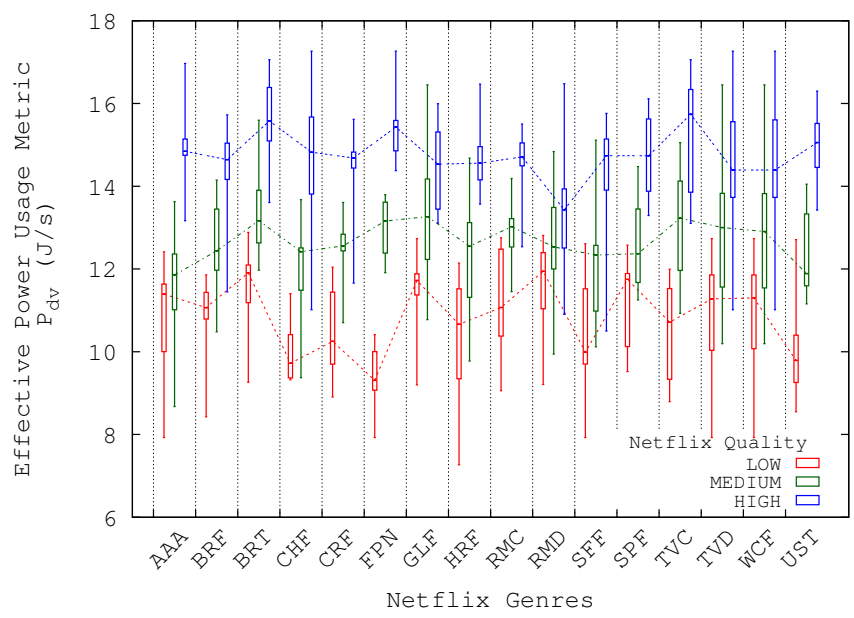

Fig. 4: Summary energy usage for several genres from the corpus of Netflix videos at the available quality levels. (The lines joining the boxplots are visual aids only, showing variation in median values.)

(e.g [3] and [13]) have shown that the CPU is typically the component that consumes the most significant proportion of energy in a system (disregarding the display). Other system components, such as memory and the network interface cards, also contribute to the overall energy usage to a much smaller (but not negligible), extent [13].

\section{The effect of hardware decoding}

We also investigated the effects of hardware assisted decoding on energy usage. While most modern processors will have some form of graphics capabilities e.g MMX, SSE, QuickSync (on Intel chipsets), which allow them to playback video efficiently, some systems will also have a dedicated Graphics Processing Units (GPU). GPUs are often intended to support 3D rendering e.g. for gaming, but often also support video playback. The power usage for hardware-assisted video playback compared with that done solely using the CPU are shown in Figure 5. The insertion of our graphics card added $22 \mathrm{~W}$ to the idle power of our desktop system (up from $78 \mathrm{~W}$, to $100 \mathrm{~W}$ with the card installed). This means that the system consumed much more energy even while idle.

We did not observe any improvements in energy usage for video playback due to the GPU. This might be due to the Linux environment in which we ran our experiments, in which Silverlight is not natively supported by the GPU drivers. However, the key takeaway point from this is that graphics cards which are optimised for 3D and games support, may not function properly for video, while adding extra idle power consumption to the system. Indeed, modern graphics cards may add upto $\sim 40 \mathrm{~W}$ to system idle power ${ }^{2}$, but the mean decoding power with CPU only is typically less than this on our testbed (See Table II).

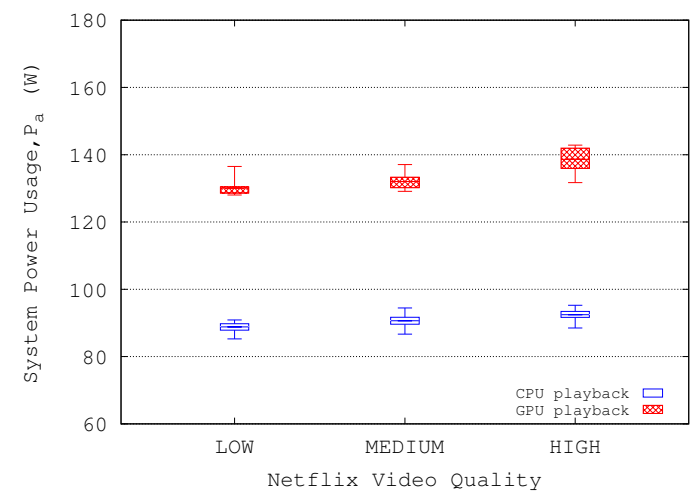

Fig. 5: System wide power usage for video playback using a CPU vs using a GPU. The GPU adds considerable idle power to the system, but does not offer any gains in efficiency.

\section{E. Network usage and energy implications}

The network usage has energy implications not just on an individual client system (more detail in Section V), but on endto-end delivery. All our experiments were performed within an office environment at the University of St Andrews, Scotland, UK. However, almost all the video traffic originated from Netflix servers located in London, England, UK, $\sim 725 \mathrm{Km}$ ( $\sim 50$ miles) away (with some small levels of signalling traffic from AWS servers in Luxembourg and Northern Ireland). Using traceroute, we can see that this traffic had to traverse an average of 11 hops, using energy at all the network devices and systems along the path.

In Figure 3c, we see that the bit rate for HIGH quality (mean $5.98 \mathrm{Mbps}$, maximum: $8.3 \mathrm{Mbps}$ ) is $\sim 2.4$ times more than for

\footnotetext{
${ }^{2}$ Tom's Hardware http://goo.gl/sWUpyF
} 
LOW quality (mean: 2.39 Mbps, maximum: 2.97 Mbps) for our experimental runs. In Figure 6, we show a snapshot of bitrates sampled over each second for the playback of one video title: 'House of Cards S1E1'. This graph highlights Netflix's default streaming behaviour [14]; a large playback buffer is filled at the beginning of a streaming session and then smaller chunks of video are downloaded at intervals as viewing progresses.

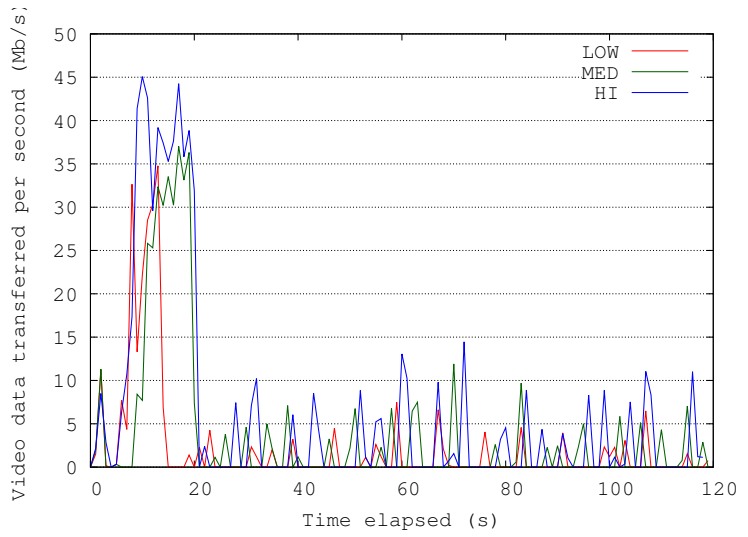

Fig. 6: Snapshot of bitrates for playback a single video, 'House of Cards (S1E1)', at each Netflix quality level.

We briefly investigated the effect of just the network traffic on the energy consumption at the client. We used iperf to investigate the energy usage of the system due to the NIC. This tool is relatively lightweight; during this test, less than $4 \%$ of the CPU and negligible (0-1\%) of the memory is used. We found that at the range of bit-rates observed during streaming from Netflix (1 Mbps to $60 \mathrm{Mbps}$ ), the power used by the system varies by $\sim 1 \mathrm{~W}$. So, for our client system, the total energy used is dominated by the energy for processing video playback by the CPU when compared to the NIC.

\section{ENERgy SAVIngS AND TRAde-OFFS}

We make estimates on what our observations might translate to when extrapolated to VoD usage at global scales. These estimates are all in the spirit of a Fermi estimate, in order that the research community can understand the significance of impacts from even small savings at individual client systems.

\section{A. Estimating energy savings by user devices}

In Section IV, we observed that different videos and genres can have widely varying energy usage profiles even at the same quality level, due to the different spatial and temporal characteristics of these videos. For example, a certain video / genre (such as a drama or documentary), may typically have fewer screen changes or inter-frame motion than, say, intense action films. These characteristics will have an impact on how much CPU, and thus energy, is required to playback a given video. This is clear in Figure 3a, where we observe that some videos which are LOW quality actually consume as much energy as other videos which are MEDIUM and HIGH quality.

Overall, we do observe a discernible trend in the difference in the median values for energy (and other system resource usage) with values of $10.8 \mathrm{~J} / \mathrm{s}_{v}, 12.8 \mathrm{~J} / \mathrm{s}_{v}$ and $14.6 \mathrm{~J} / \mathrm{s}_{v}$ for $P_{d v}$ at the three available quality levels on Netflix over the whole corpus. Considering that $J / s_{v}$ is equivalent to the Watt (W), these differences of $\sim 2 J / s_{v}$ between each quality level, might not seem significant for a single system or user.

However, the impact of these differences at a global scale are significant. According to Sandvine [1], the top $15^{\text {th }}$ percentile of Internet Users in North America are 'cord- cutters' - users who no longer have normal TV subscriptions and watch all their content via the Internet. These users consume $\sim 100$ hours of video per month. So, for such a single Netflix subscriber, with the assumption that the energy difference on their equipment will be similar to our measurements, we can estimate the energy impact of their viewing habits.

Based on our measurements, the savings possible for this user by watching all content in LOW quality instead of HIGH quality would be approximately $3.7 \mathrm{~J} / \mathrm{s}_{v}$ (Joules per second of video playback). For 100 hours of video a month, that sums to $1,332 \mathrm{KJ}(0.37 \mathrm{KWh})$ per month or $15,946 \mathrm{KJ}(4.43 \mathrm{KWh})$ per year. Superficially, this amount of energy appears negligible, so it may be difficult for the individual user to rationalise a decision to watch video at the LOW quality level. Note that this figure does not consider the idle power of the devices used to watch video, just the extra energy required for playback.

Let us scope our Fermi estimate to a global scale. Netflix have claimed that they have over 50 million subscribers ${ }^{3}$, and Nielsen ${ }^{4}$ suggests that roughly $60 \%$ ( $\sim 30$ million) of Netflix subscribers watch via a personal computer [6]. (As Netflix continues to expand internationally, this figure is likely to continue to grow.)

Netflix have further claimed that 2 billion hours of video are streamed from their servers every month. For the purpose of this estimate, we shall assume that all this video is streamed to equipment that is similar to ours. Again, at savings of $3.7 \mathrm{~J} / s_{v}$ by watching all video at LOW quality instead of $\mathrm{HIGH}$, this would be a total of $\sim 7.4$ million KWh a month or 88.8 million KWh a year. To appreciate the scale of these savings, we shall make an assessment of the impact of what this energy represents in various parts of the world. If these savings where achieved, this would be enough energy required to power 21,382 homes in the United Kingdom or 114,138 homes in India for a year (based on available estimates) - see the summary in Table III, which also presents estimates of $\mathrm{CO}_{2}$ emission savings that are possible in different parts of the world.

Of course, this is by no means intended as an authoritative or comprehensive estimate, and that is not the aim of this exercise. We simply wish to show the sheer scale of the energy savings potentially possible, and raise awareness of the amount of energy consumed by video streaming. For example, we have made the generalisation that all users have equipment that has similar performance to our testbed and will see similar differences in energy usage. This may not be the

\footnotetext{
${ }^{3}$ http://ir.netflix.com

${ }^{4}$ http://www.nielsen.com
} 


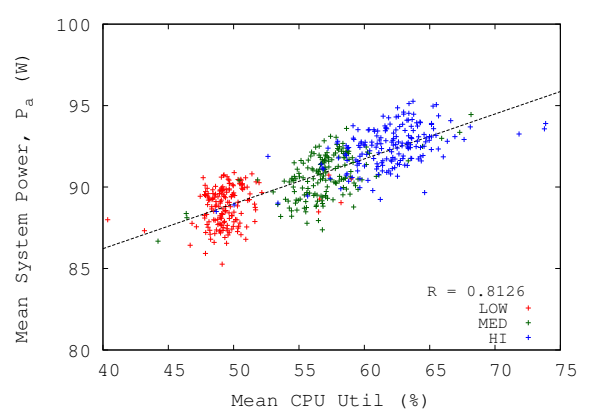

(a) Correlation between CPU usage and power $(\mathbf{R}=\mathbf{0 . 8 1 6 2})$ quality levels (202 videos). Each data point is the mean of 5 runs, each run is 120 seconds). Memory and bitrate correlations are deceptive

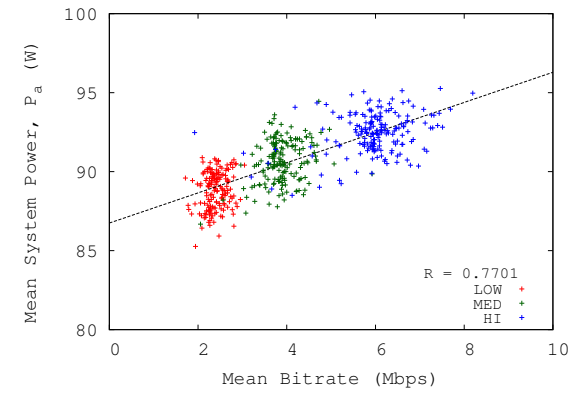

(c) Correlation between mean bitrate and power $(\mathbf{R}=\mathbf{0 . 7 7 0 1})$
TABLE III: Estimate of potential annual impact of 88.8GWh savings by user devices based on 24 billion hours of Netflix video playback.

\begin{tabular}{|l|c|c|c|c|}
\hline Country & $\begin{array}{c}\text { Mean KWh } \\
\text { /home }\end{array}$ & No of homes & Cost $^{b}$ (US\$) & $\mathrm{KgCO}_{2}{ }^{c}$ \\
\hline India & 778 & 114,138 & $710 \mathrm{M}$ & $34.2 \mathrm{M}$ \\
\hline Nigeria & 604 & 129,824 & $1,598 \mathrm{M}$ & N/A \\
\hline UK & 4153 & 21,382 & $1,770 \mathrm{M}$ & $15.9 \mathrm{M}$ \\
\hline US & 11789 & 7,532 & $1,065 \mathrm{M}$ & $19.9 \mathrm{M}$ \\
\hline \multicolumn{5}{|c}{$\begin{array}{c}{ }^{a} \text { From World Energy Council Data for } 2011^{5} \\
{ }^{b} \text { From various local electricity boards. }\end{array}$} \\
\\
${ }^{c}$ http://www.wec-indicators.enerdata.eu/
\end{tabular}

case as some users may make use of more (or less) efficient computing hardware, and devices will have larger differences in energy usage. We have made use of mid-range, inexpensive commodity hardware in our experiments, of a class which might be used by a large proportion of every day Internet users. We have also made these estimates solely based on published statistics for Netflix. There are several other VoD services which have very significant numbers of users and viewing hours, so our numbers are likely to be an underestimate of the overall impact of VoD.

\section{B. Estimating energy savings in the network}

Transcoding of Netflix's content is done using cloud-based infrastructure, with a single title reportedly encoded into $\sim 50$ formats. In our previous work [7], we observed significant differences in energy usage in encoding different codecs, up to 3 orders of magnitude depending on picture settings and codecs. However, this encoding would typically take place in specialized, energy-efficient data centres, along with several other workloads. In this kind of environment, energy usage would be highly controlled and the scope for energy savings may be smaller. Investigating energy-efficient video transcoding is an avenue for future work.

The Internet is extremely heterogeneous in nature, so the various links that the video data traverses will be under different administrative domains, using different media, from fibre optic links, to copper, to wireless, and each having varying energy costs and carbon emissions. A considerable amount of research has investigated the energy usage at the network level, and how this is affected by several different parameters such as the network media, protocol, and bit-rate etc. For instance, some authors ([15] [16]) have estimated that the network infrastructure of the Internet consumes up to $2 \%$ of the World's generated electricity, corresponding to approximately $420 \mathrm{TWh}$ per year, with variable energy consumption rates observed between $23 \mathrm{KWh}$ to $109 \mathrm{KWh}$ per year per subscriber [17]. Current energy consumption is due to an estimated 600 exabytes of Internet traffic (2013 estimate), which is expected to triple by 2018 [2], of which, as stated previously, an increasing proportion is video.

Other studies have also investigated energy consumption at the network to the bit level. Baliga et al. [18] estimate that the energy consumption of data transmitted over the Internet is $2-4 \mu \mathrm{J} / \mathrm{b}$ at higher access rates ( $<100 \mathrm{Mbps})$, to $75 \mu \mathrm{J} / \mathrm{b}$ at lower access rates. Similarly, in our previous work [19], we investigated how various wireless access networking technologies (802.11) can consume a varying amount of energy at the client, varying between $0.5-1.1 \mu \mathrm{J} / \mathrm{bit}$ for large packet sizes to $2.3-10 \mu \mathrm{J} / \mathrm{b}$ for smaller packet sizes.

Extending our Fermi estimation exercise from Section V-A, the figures for data transmitted per second at the various quality levels are shown in Figure $3 \mathrm{c}$ and discussed in Section IV-E. We can extrapolate to a global scale using these values. Firstly, let us estimate the energy usage for a single cordcutter who views 100 hours of Netflix video every month. Using the upper bound of Baliga et al's estimated values [18] of energy usage on wide area networks $(4 \mu \mathrm{J} / \mathrm{b})$, and based on our measurements for bitrates, we can estimate the amount of data required for 100 hours of Netflix video at the different quality levels. For LOW, MEDIUM and HIGH quality, a single user consumes $0.115 \mathrm{KWh}, 0.192 \mathrm{KW}$ and $0.289 \mathrm{KWh}$ per year, respectively. Furthermore, since we are informed that 24 billion hours of video are streamed from Netflix yearly, we can estimate the amount of energy that would be used annually for the network globally as $27.64 \mathrm{GWh}, 44.92 \mathrm{GWh}$ and $68.78 \mathrm{GWh}$, for LOW, MEDIUM and HIGH quality respectively. We can see that there is a difference of 41.14 GWh between playing LOW quality and HIGH quality Netflix video in terms of network usage. The impacts of this energy usage is summarised in Table IV using the UK as an example. 
TABLE IV: Estimate of potential annual impact of energy usage in the network based on 24 billion hours of streamed Netflix video

\begin{tabular}{|l|c|c|c|c|}
\hline Quality & $\begin{array}{c}\text { Mean bitrate } \\
(\mathrm{Mbps})\end{array}$ & $\mathrm{KWh}$ & $\mathrm{Cost}^{a}$ & $\mathrm{KgCO}_{2}{ }^{b}$ \\
\hline LOW & 2.40 & $27.64 \mathrm{M}$ & $\mathrm{GB} £ 4.14 \mathrm{M}$ & $4.70 \mathrm{M}$ \\
\hline MEDIUM & 3.90 & $44.92 \mathrm{M}$ & $\mathrm{GB} £ 6.72 \mathrm{M}$ & $7.62 \mathrm{M}$ \\
\hline HIGH & 5.98 & $68.78 \mathrm{M}$ & $\mathrm{GB} £ 10.30 \mathrm{M}$ & $11.68 \mathrm{M}$ \\
\hline \multicolumn{4}{|c|}{${ }^{a}$ From [20], mean cost of GB $£ 577$} & year per household. \\
${ }^{b}$ UK government data, 10 May $2014,0.17 \mathrm{KgCO}_{2} / \mathrm{KWh}^{6}$.
\end{tabular}

\section{Limitations and future developments}

For this study, we have only considered a single desktop hardware configuration. We are conscious that other hardware (and even software) might have very different energy usage patterns. The hardware we use is, however, cheap and widely available, and represents broadly the class of hardware that many end users will use. Furthermore, we only make estimates of energy use based on $P_{d v}$, which is the effective (extra) energy required to playback video, disregarding the idle power of the system. This helps in de-biasing our estimates from a single hardware configuration, but might underestimate the overall energy consumption for a device that is dedicated to video playback only.

We have also not considered mobile devices in our experiment and estimates. Modern mobile devices usually have hardware assistance for video playback, with special chipsets which can decode video efficiently, albeit for a limited set of video formats, e.g. H.264, MPEG-2, MPEG-4. Some popular manufacturers have partnered with Netflix for ensuring such device-based support. Personal and anecdotal experience shows that watching video on a mobile device drains the battery much faster than during normal usage. This is another interesting avenue for future work. In any case, the bulk of Netflix content is still watched on non-mobile systems.

For our experiment, we have only considered video up to a maximum resolution of $1080 \mathrm{p}$ (HIGH quality). $4 \mathrm{~K}$ video (also known as 4096p or Ultra High Definition (UHD) TV) is the most recent picture size available, with incremental hardware support in CPUs, displays and smart TVs. At the time of writing (early 2015), Netflix only supports $4 \mathrm{~K}$ playback on a small subset of smart $\mathrm{TVs}^{7}$ ). However, as networks become more capable, and $4 \mathrm{~K}$-capable devices become available, then the energy usage of $4 \mathrm{~K}$ video will become more significant, especially if the calculations we have made here scale linearly for $4 \mathrm{~K}$.

As we can see from Figure 2, Netflix states that UHD requires more than double the amount of data for regular $\mathrm{HD}$, and so there will be a proportionate increase in energy consumption and carbon emissions, both in the network and at client devices. This is why it is very important for users and developers to be made aware of the implications of their video streaming habits.

\footnotetext{
${ }^{6} \mathrm{http} / / /$ www.rensmart.com/Information/KWHToCO2Conversion

${ }^{7}$ https://help.netflix.com/en/node/13444
}

\section{Playback Settings}

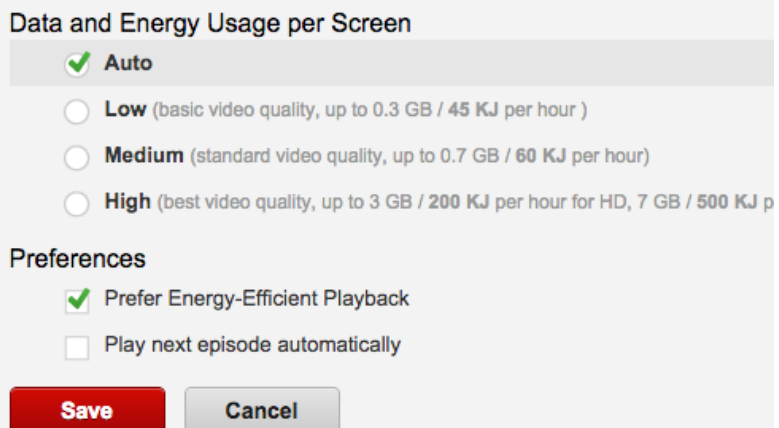

Fig. 8: A mock-up of a Netflix quality selection page with very simple energy usage information (compare to Figure 2). This information could be based on a system specific benchmark or crowdsourced usage data.

\section{ENABLING USER CHOICE}

Ultimately, for energy savings to be made, users have to make choices. We need to make users (i) aware of energy usage; and then (ii) encourage them to make conscious efforts to reduce energy usage. Demand Side Management (DSM) techniques are often used within the power industry to reduce consumers' demand for energy by encouraging behavioural change through education, information and incentivisation. In previous work [5], we found that ICT system users within a lab environment who were given feedback on their energy usage - and incentives to save energy - were able to reduce energy usage by up to $56 \%$ (for a single user). A mean $16 \%$ reduction overall was observed across the test group. If digital video users are well-informed about the potential energy usage of their habits, e.g. presented with information about energy usage and consumption, they may be encouraged to reduce energy usage.

\section{A. Informing the user}

Previously [7], we introduced the concept of a dynamic energy label for video which would inform a user about the resource usage of the video they are about to watch. This information would be generated based on results from a benchmarking exercise. Based on this information, a user could choose to watch a less resource-intensive video. Figure 8 is a similar concept; a mock-up of a modified Netflix quality selection page (as shown in Figure 2), which presents simple energy usage information to users.

\section{$B$. Incentives and a question of quality}

Netflix users pay higher subscription fees per month to be able to access HD (+GB£1) and UHD (+GB£3) content in the $\mathrm{UK}^{8}$. Thus, it would be difficult for these subscribers to rationalise the conscious decision to watch lower quality video to save energy (thus wasting the extra money paid for subscription). However, we can look at this from another angle. Netflix is implicitly 'incentivising' subscribers

\footnotetext{
${ }^{8}$ https://www.netflix.com/getstarted?locale=en- GB
} 
to watch lower quality video by offering lower subscription charges. This could be made more explicit. This will mean using lower computing resources and energy, and lowering carbon emissions on client systems, servers and networks. The incentive in this case is financial, but also some users may be incentivised additionally by environmental concerns. We have already estimated the impact of these differences on client devices and on the network in Section V. A costbenefit analysis of the implications for Netflix and other VoD providers would be an interesting exercise for future work, to see if such an approach is viable: will the reduced revenue from subscriptions be offset by the savings in energy usage by providers?

Indeed, in our ongoing work, we have found that there is little difference in quality of experience (QoE) between HD (1080p) and UHD (2160p), at least using objective measures such as the Y-SSIM metric [21]. This needs further investigation to enable energy-cost-quality trade-offs and incentives to be defined and made available to users.

Other services like YouTube and BBC iPlayer, could use various other types of incentives. For example, YouTube, which is an entity within the wider Google ecosystem, could perhaps offer some form of 'green kudos' points, perhaps on Google+ or on other social media networks, showcasing the user's environmental consciousness to friends and followers. Incentives to save energy at the client-side while using ICT is an interesting area for future work.

\section{CONCLUSION AND FUTURE WORK}

We presented our assessment of the energy usage and carbon footprint of Video-on-Demand ( $\mathrm{VoD}$ ), using the popular Netflix service as a case study. Netflix provides videos in 3 quality settings, LOW, MEDIUM and HIGH. We found, from measurements on 202 Netflix videos across several genres that there is a clear mean difference between the lowest and highest quality of $\sim 3.7 \mathrm{~J} / s_{v}\left(1 \mathrm{~J} / s_{v}\right.$ is equal to 1 Watt). While this difference or possible savings may appear small for a single client, an estimate on a global scale show just how significant this energy usage actually is for a population of VoD users. We found that there is a possible difference of $88.8 \mathrm{GWh}$ a year, enough to power over 20000 homes in the UK or 100000 homes in India. We also briefly investigated the relationship between the various Netflix genres and energy usage, and the effect of hardware-assisted decoding. Finally, we discussed ways in which users may be empowered to make the conscious decisions to save energy while using digital video services like Netflix.

We have identified several avenues for future work. These include: a finer-grained analyses of the energy usage of video playback across genres / spatio-temporal classification; expanding considerations to mobile and other devices (e.g Smart TVs); exploring green QoE metrics and energy usage feedback for video; and user incentives for saving energy while using digital video. A benchmark tool for video energy usage, based partly on the work presented here, is currently under development.

\section{ACKNOWLEDGEMENTS}

This work was partly supported by the IU-ATC project, funded by grant EP/J016756/1 from the Engineering and Physical Sciences Research Council (EPSRC). Oche Ejembi is funded by the Scottish Informatics and Computer Science Alliance (SICSA).

\section{REFERENCES}

[1] "Global Internet Phenomena Report 1H 2014,” Jul 2014. [Online]. Available: http://goo.gl/Lb33Wf

[2] Cisco Systems, "Cisco Visual Networking Index 2014," Jun 2014. [Online]. Available: http://goo.gl/3gZy0z

[3] P. Somavat, S. Jadhav, and V. Namboodiri, "Accounting for the Energy Consumption of Personal Computing Including Portable Devices," in e-Energy2010: 1st ACM Intl. Conf. Energy-Efficient Computing and Networking, 2010.

[4] J. Pan, R. Jain, and S. Paul, "Nine lessons learned from a green building testbed: A networking and energy efficiency perspective," in WCST 2013 - World Congress on Sustainable Technologies, Dec 2013.

[5] Y. Yu and S. N. Bhatti, "The Cost of Virtue: Reward As Well As Feedback Are Required to Reduce User ICT Power Consumption," in e-Energy 2014: 5th ACM Intl. Conf. on Future Energy Systems, Jun 2014.

[6] Nielsen Research, "Binging is the new viewing for over-the-top streamers," Aug 2013. [Online]. Available: http://goo.gl/0uEEFC

[7] O. Ejembi and S. N. Bhatti, "Help save the planet: Please do adjust your picture," in MM2014: 22nd ACM Intl. Conf. on Multimedia, Nov 2014.

[8] A. Shehabi, B. Walker, and E. Masanet, "The energy and greenhousegas implications of internet video streaming in the United States," Environmental Research Letters, vol. 9, no. 5, 2014.

[9] J. Ramos-munoz, J. Prados-Garzon, P. Ameigeiras, J. Navarro-Ortiz, and J. Lopez-soler, "Characteristics of mobile YouTube traffic," IEEE Wireless Comms., vol. 21, no. 1, Feb 2014.

[10] V. Adhikari, Y. Guo, F. Hao, M. Varvello, V. Hilt, M. Steiner, and Z. L. Zhang, "Unreeling Netflix: Understanding and improving multi-CDN movie delivery," in INFOCOM 2012: Proc. of 31st Annual IEEE Intl. Conf. on Computer Comms., Mar 2012.

[11] M. Hoque, M. Siekkinen, and J. Nurminen, "Energy Efficient Multimedia Streaming to Mobile Devices: A Survey," IEEE Comms. Surveys \& Tutorials, vol. 16, no. 1, Jan 2014.

[12] "UK fixed-line broadband performance, May 2014," Oct 2014. [Online]. Available: http://goo.gl/gGx9cI

[13] J. Arjona, A. Chatzipapas, A. Fernández, and V. Mancuso, "A Measurement-based Analysis of the Energy Consumption of Data Center Servers," in e-Energy 2014: 5th ACM Intl. Conf. Future Energy Systems, Jun 2014.

[14] J. Martin, Y. Fu, N. Wourms, and T. Shaw, "Characterizing Netflix bandwidth consumption," in CCNC 2013: IEEE Conf. on Consumer Comms. and Networking, Jan 2013.

[15] S. Lambert, W. V. Heddeghem, W. Vereecken, B. Lannoo, D. Colle, and M. Pickavet, "Worldwide electricity consumption of communication networks," Opt. Express, vol. 20, no. 26, Dec 2012.

[16] M. Gupta and S. Singh, "Greening of the Internet," in Proc. of the 2003 Conf. on Apps., Tech., Arch., \& Protocols for Computer Comms., 2003.

[17] "SMART 2020: Enabling the low carbon economy in the Info. age," The Climate Group, 2008. [Online]. Available: http://goo.gl/12MNKO

[18] J. Baliga, R. Ayre, K. Hinton, W. Sorin, and R. Tucker, "Energy Consumption in Optical IP Networks," Journal of Lightwave Technology, vol. 27, no. 13, Jul 2009.

[19] M. Tauber, S. N. Bhatti, and Y. Yu, "Towards Energy-Awareness In Managing Wireless LAN Applications," in NOMS 2012 - IEEE Network Operations and Mgt. Symp., Apr 2012.

[20] "UK Energy Statistics ," May 2014. [Online]. Available: http: //goo.gl/gqXSWl

[21] O. Ejembi and S. N. Bhatti, "Go Green with EnVi: The Energy-Video Index," in ISM2015: IEEE Intl. Symp. on Mulitmedia, Dec 2015. 